\title{
High frequencies of circulating memory T cells specific for calreticulin exon 9 mutations in healthy individuals
}

\author{
Morten O. Holmström (10), Shamaila M. Ahmad², Uffe Klausen², Simone K. Bendtsen², Evelina Martinenaite², \\ Caroline H. Riley ${ }^{3}$, Inge M. Svane ${ }^{2,4}$, Lasse Kjær ${ }^{1}$, Vibe Skov' ${ }^{1}$, Christina Ellervik ${ }^{5,6,7}$, Niels Pallisgaard ${ }^{8}$, \\ Hans C. Hasselbalch ${ }^{1}$ and Mads H. Andersen ${ }^{2,9}$
}

\begin{abstract}
Mutations in exon 9 of the calreticulin gene (CALR) frequently occur in patients with chronic myeloproliferative neoplasms (MPN). Patients exhibit spontaneous cellular immune responses to epitopes derived from the mutant CALR C-terminus, and CALR-mutant-specific T cells recognize autologous CALR-mutant malignant cells. This study investigated whether CALR-mutant-specific T cells occur naturally in CALRwt MPN-patients and in healthy individuals. Specific immune responses against epitopes in the mutant CALR peptide sequence were detected in both CALRwt MPN-patients and in healthy individuals. Healthy donors displayed more frequent and stronger CALR-mutant specific T-cell responses compared to the responses identified in CALR-mutant MPN-patients. Several T-cell responses were identified in healthy donors directly ex vivo. Importantly, by running functional analyses on live-sorted immune cells from healthy donors, we showed that circulating CALR-mutant-specific immune cells are T-memory cells. These findings suggest, that healthy individuals acquire a CALR exon 9 mutation, but the immune system reacts and clears the mutant cells, and during this reaction generates CALR-mutant specific T-memory cells. We believe that these findings provide the evidence for tumor immune surveillance in MPN.
\end{abstract}

\section{Introduction}

In 2013, two independent research groups reported on the occurrence of somatic mutations in exon 9 of the calreticulin $(C A L R)$ gene in $75 \%$ of patients with Janus kinase $2(J A K 2)$ wt chronic myeloproliferative neoplasms $(\mathrm{MPN})^{1,2}$. We recently provided evidence that patients with CALR-mutant MPN exhibit frequent, spontaneous cellular immune responses against epitopes derived from the mutant CALR C-terminus ${ }^{3}$, and that CALR-mutantspecific $T$-cells derived from patients can recognize and kill autologous CALR-mutant cells in a CALR-mutant dependent manner ${ }^{4}$. As such the CALR mutations

\footnotetext{
Correspondence: Morten O. Holmström (holmeren1@yahoo.dk) ${ }^{1}$ Department of Hematology, Zealand University Hospital, Roskilde, Denmark ${ }^{2}$ Center for Cancer Immune Therapy, Department of Hematology, Copenhagen University Hospital Herlev, Herlev, Denmark Full list of author information is available at the end of the article.
}

generate an immunogenic antigen that could be used as target for cancer immune therapy ${ }^{5}$.

As the CALR mutations are only identified in patients with myeloid cancer, the immune system in healthy individuals should not have been challenged with mutant CALR epitopes, and hence should not react to stimulation with these. Accordingly, several studies on immune reactivity against other shared commonly occurring mutations, such as $p R A S$ mutations and $B R A F$ mutations in solid cancers, have indicated that cells from healthy individuals fail to show spontaneous immune responses against these neoantigens ${ }^{6,7}$. In regard to immune responses to the BCR-ABL fusion transcript, one study showed responses in peripheral blood mononuclear cells (PBMC) from 3/18 healthy donors after stimulation in vitro with autologous dendritic cells that had been pulsed with a BCR-ABL peptide ${ }^{8}$, whereas another study 
failed to show immune responses against the transcript in healthy individuals ${ }^{9}$. Other studies have focused on immune responses against the somatic MYD88L265P mutation, which occurs in several types of lymphoma ${ }^{10}$. One study demonstrated a T-cell response, when T-cells derived from healthy individuals were stimulated with the MYD88L265P epitope, but the responses required two stimulations with peptide-pulsed dendritic cells ${ }^{11}$.

Of interest, none of the above studies have demonstrated immune responses against tumor-specific antigens in cells from healthy individuals tested in an ex vivo setting, without prior in vitro peptide stimulation. Even more, it has never been shown, which cells in the T-cell compartment actually harbor these neo-antigen-specific $\mathrm{T}$ cells.

Given the high immunogenicity of the CALR exon 9 mutations we investigated if healthy donors display $\mathrm{T}$-cell responses specific for the CALR mutations and if so, whether such CALR-mutant specific $\mathrm{T}$ cells are antigen experienced $\mathrm{T}$-memory cells $\left(\mathrm{T}_{\mathrm{mem}}\right)$ or naive $\mathrm{T}$ cells $\left(\mathrm{T}_{\text {naive }}\right)$. The identification of a memory response is important, as CALR-mutant specific $T$ cells in the $T_{\text {mem }}$ compartment suggest that healthy donors may acquire a CALR exon 9 mutation, which is cleared by specific Tcells and $\mathrm{T}_{\mathrm{mem}}$ is established in the process.

This study demonstrates that healthy donors display stronger and more frequent CALR-mutant specific T-cell responses compared to CALR-mutant patients. Even more, we show that the mutant CALR C-terminus harbors an immunogenic hotspot, and finally that CALRmutant specific $\mathrm{T}$ cells in healthy individuals are $\mathrm{T}_{\mathrm{mem}}$.

\section{Materials and methods Materials}

Buffy coats from anonymized blood donors were acquired from the blood bank at Rigshospitalet, Copenhagen, Denmark. Peripheral blood mononuclear cells (PBMC) from patients with JAK2V617F-mutant MPN were acquired after informed consent from the patient. PBMC were isolated with Lymphoprep (Axis Shield, Oslo, Norway) and frozen in fetal calf serum with $10 \%$ dimethyl sulfoxide (Sigma-Aldrich, St. Louis, MO, USA). To determine whether age might influence specific immune responses to $C A L R$-mutants, we isolated PBMCs from 50 healthy individuals of different ages. All participants provided informed consent, in agreement with the Helsinki Declaration, before study entry. The project was approved by the local Ethics Committee of the Zealand Region (Approval number SJ-175 and SJ-456).

\section{Peptides}

We chose to work with the following peptides, which were either provided by KJ Ross-Petersen (Klampenborg, Denmark) or Schafer-N (Copenhagen, Denmark):
CALRLong1 (RRMMRTKMRMRRMRRTRRKMRRKMS PARP), CALRLong2 (TRRKMRRKMSPARPRTSCREACLQGWTEA), CALRLong3 (KMRMRRMRRT RRKMRRKMS), and CALRLong4 (RRMRRTRRKMR RKMSPARPRTSCREACLQGWTEA). In addition, we used the CALRLong36 (RMRRMRRTRRKMRRKM SPARPRTSCREACLQGWTEA) peptide, provided by PolyPeptide Laboratories (Strasbourg, France). We also chose to work with the following short peptides, all provided by KJ Ross-Petersen (Klampenborg, Denmark): CALR-01 (RTRRKMRRK), CALR-02 (RTSCREACL), CALR-03 (RTKMRMRRM), and CALR-04 (RTRRKMRRKM). The sequences of peptides and their relation to the entire CALR-mutant $\mathrm{C}$-terminal are given in Fig. 1. To identify the immunogenic hotspot in the mutant CALR C-terminus, we segregated the entire mutant $\mathrm{C}$-terminus into nonamer epitopes, with eight overlapping amino acids. Thus, we generated a total of 36 nonamer peptides, which we termed the CALR library (Supplementary Material 1). These epitopes were acquired from Pepscan (Lelystad, Netherlands). Negative control peptides were generated by scrambling the CALRmutant peptide sequence. We generated three negative control peptides with the following sequences: MRRTMMMMMPRRRRRRKRRSKTRAPRMRK, MERKMAEQRPCRKPSTRALATCRRWGSRT, and TSMMRRRRRRKRRKMMKRM. These peptides were provided by KJ Ross-Petersen.

\section{Enzyme-linked immunospot assay}

To analyze $C A L R$-mutant-specific immune responses, we employed Enzyme-Linked ImmunoSPOT (ELISPOT) assays, according to protocols described previously ${ }^{12}$. Immune responses were analyzed with the interferongamma (IFN- $\gamma$ ) or tumor necrosis factor alpha (TNF- $\alpha$ ) ELISPOT assay. Unless otherwise stated, all experiments were performed with the in vitro IFN- $\gamma$ ELISPOT assay. The ELISPOT assays employed 1.3 to $4 \times 10^{5}$ cells/well. Unless otherwise stated, all ELISPOT experiments were performed in triplicates. Additionally, when not otherwise stated, ex vivo ELISPOTs were performed with thawed PBMCs that had been allowed to rest overnight at $37^{\circ} \mathrm{C}$ in an incubator with $5 \% \mathrm{CO}_{2}$ and a humidified atmosphere. ELISPOT experiments were set-up as previously described $^{12}$. ELISPOT plates were analyzed and counted using the ImmunoSpot S6 Ultimate Analyzer (CTL Analyzers, Shaker Heights, OH, USA).

For the identification of immune responses against epitopes in the CALR library, we deviated from the standard in vitro stimulation protocol. For cultures that we planned to analyze for responses against the B1-C6 region of the CALR-mutant C-terminus, we performed in vitro stimulations with the CALRLong1 peptide. For cultures that we planned to analyze for response against 


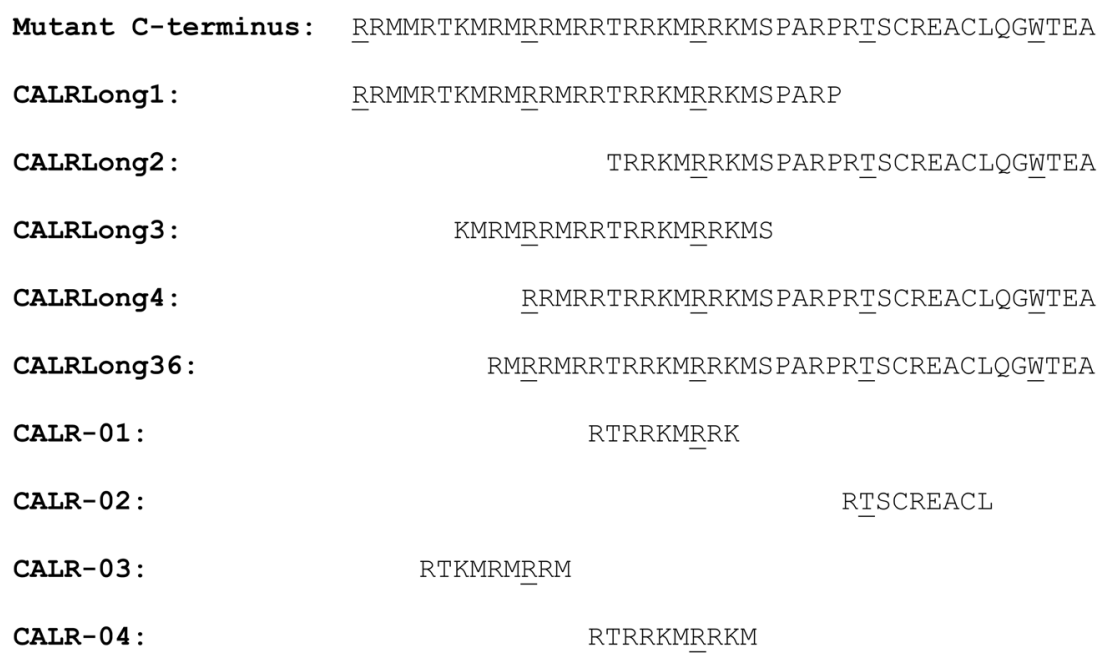

Fig. 1 Amino acid sequences for the long and short epitopes used in this study. The sequences are shown relative to the mutant CALR Cterminus. Every 10th amino acid is underscored to facilitate tracking

the C7-D12 region of the CALR-mutant C-terminus, we performed in vitro stimulation with the CALRLong2 peptide. The in vitro assays with CALRLong1 and CALRLong2 saved time and cells, with minimal effects on the sensitivity of the experiments, because CALRLong1 spanned residues B1-C6, and CALRLong2 spanned residues C7-D12.

\section{Magnetically activated cell sorting (MACS)}

$\mathrm{CD} 4{ }^{+} \mathrm{T}_{\text {mem }}$ cells and $\mathrm{T}_{\text {naive }}$ were sorted with the MACS method, with the Memory $\mathrm{CD}^{+}{ }^{+} \mathrm{T}$-cell Isolation Kit (order no 130-091-893) and with the Naive Pan T-cell Isolation Kit (order no 130-097-095), respectively (both Miltenyi Biotech, Bergish Gladbach, Germany), according to the manufacturer's protocols. Cells were thawed and rested overnight, then sorted the next day. Cells were sorted twice on a magnetic column to ensure high purity in the enriched cells. During $T_{\text {naive }}$ isolation, we added the optional anti-TCR $\gamma / \delta$ antibodies to ensure depletion of $\gamma /$ $\delta$ T cells. We analyzed the purity of the enriched fractions by staining with Fixable Viability Stain 510, CD3-APCH7, CD45RO-PE, and CD45RA-APC (all BD Biosciences, San José, CA, USA). Cells were analyzed on a FACS Canto II flow cytometer (BD Biosciences, San José, CA, USA) equipped with FACS Diva Software. Gating strategy for the identification of $\mathrm{CD}^{+} \mathrm{T}$ cells for the phenotypic analysis of memory cell markers is provided in Supplementary Material 2.

\section{Fluorescence-activated cell sorting (FACS) of live cells}

Cells were thawed, and then rested overnight in $\mathrm{x}$-vivo medium (Lonza, Basel, Switzerland) with 5\% human serum in an incubator. The next day, cells were washed twice in FACS buffer, then stained with the following:
LIVE/DEAD Fixable Near-IR Dead Cell Stain Kit (Waltham, MA, USA), CD45RA-APC, CD62L-PE, and CD3FITC (BD Biosciences, San José, CA, USA). Cells were stained for $30 \mathrm{~min}$ on ice, washed twice, and then resuspended in FACS buffer at $50 \times 10^{6}$ cells $/ \mathrm{mL}$. Next, cells were sorted on a FACS ARIA flow cytometer with appropriate application settings and compensation controls. Cell sorting was performed with a purity setting. After sorting, cells were allowed to rest in $\mathrm{x}$-vivo medium (Lonza, Basel, Switzerland) in an incubator. After sorting of all the cells, samples of $10^{4}$ cells were analyzed to ensure proper sorting purity. Gating strategy for the identification of $\mathrm{CD}^{+} \mathrm{T}$ cells for the phenotypic analysis and subsequent sorting of different memory cell fractions is provided in Supplementary Material 2. Materials and methods for intracellular cytokine staining (ICS), establishment of $\mathrm{T}$-cell cultures specific for mutant CALR epitopes and statistical analyses are provided in Supplementary Material 3.

\section{Results}

Patients with JAK2V617F-mutant MPN harbor an immune response to mutant CALR epitopes

We scrutinized peripheral blood mononuclear cells (PBMC) from patients with JAK2V617F-mutant polycythemia vera $(\mathrm{PV})$ for immune responses against the immunogenic CALRLong1 and CALRLong2 epitopes that are derived from the mutant CALR C-terminus ${ }^{3}$. Surprisingly, 2/6 patients with $\mathrm{PV}$ exhibited a significant response against CALRLong1 (data not shown) and 3/5 had a significant immune response against CALRLong2 (Fig. 2a), according to the distribution free resampling method (DFR) ${ }^{13}$. CALR/JAK2 double mutants are very rare and these mutations are generally mutually exclusive ${ }^{14-17}$. 


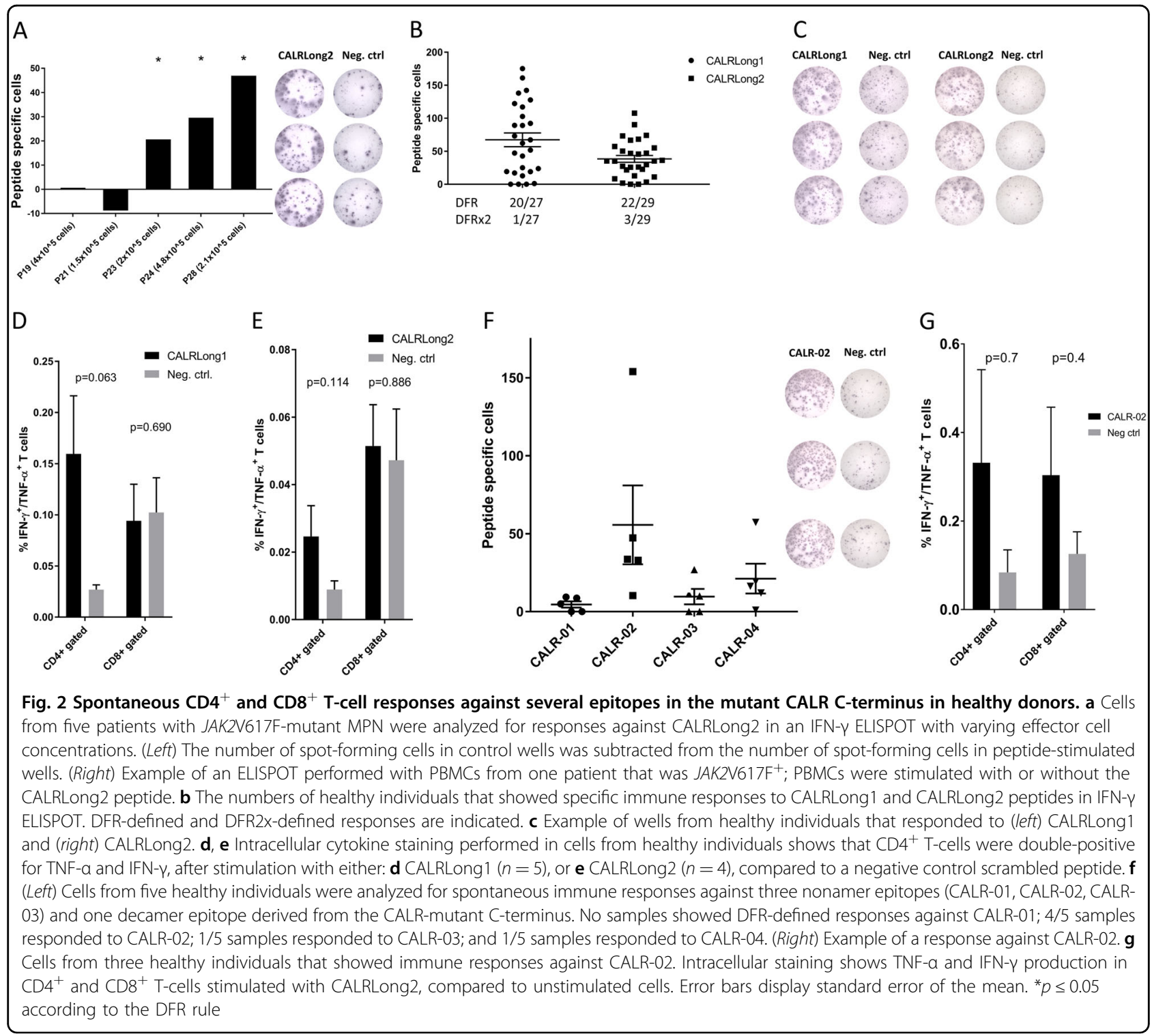

Cells from healthy individuals display strong and frequent immune responses against the CALRLong1 and

\section{CALRLong2 peptides and against short CALR-mutant epitopes}

Next, we investigated whether cells from healthy individuals might also exhibit an immune response against the CALRLong1 and CALRLong2 epitopes. Surprisingly, cells from 20/27 (74\%) individuals showed a significant response to CALRLong1 (Fig. 2b, c), and cells from 22/29 (76\%) individuals showed a significant immune response to CALRLong2 (Fig. 2b, c). Using ICS, 5 and 4 healthy individuals were analyzed for responses against CALRLong1 (Fig. 2d) and CALRLong2 (Fig. 2e), respectively. Stimulation of PBMC with mutant CALR epitopes lead to increased production of TNF- $\alpha$ and IFN- $\gamma$ from CD4 ${ }^{+} \mathrm{T}$ cells. The responses were only borderline significant, probably due to low sample size.

Given these highly surprising results, we investigated the immunogenic potential of three CALR-mutant nonamer epitopes (CALR-01, CALR-02, and CALR-03) and one CALR-mutant decamer epitope (CALR-04). CALR-02 induced responses in cells from $4 / 5$ individuals, and CALR-03 and CALR-04 induced responses in cells from one individual each (Fig. 2f). Furthermore, we tested cytokine release in three donors against CALR-02 using ICS (Fig. 2g). However, the difference in cytokine release between stimulated and unstimulated cells did not reach statistical significance. 
Screening for immune responses against nonamer epitopes in the mutant CALR C-terminus identifies an immunogenic hotspot sequence

Given the remarkably high frequency of responses against the CALR-mutant epitopes we verified that there was no homology between the 44 amino acid mutant CALR sequence and other known epitopes by the Basic Local Alignment Search Tool (BLAST) ${ }^{18}$ using the blastpprogramme and the non-redundant protein sequences (nr) database. We next examined whether the CALRmutant specific immune responses might be directed towards a certain part of the mutant sequence. Hence, we divided the 44-amino acid mutant $\mathrm{C}$-terminus that is shared between the majority of CALR-mutant patients, into nonamer epitopes, with eight overlapping amino acids (Supplementary Material 1). Accordingly, we generated 36 nonamer epitopes, and analyzed PBMCs from ten healthy individuals for immune responses against each of these epitopes. We observed immune responses against all parts of the mutant CALR sequence (Supplementary Material 4); however, we could clearly identify an immunogenic hotspot located in the $\mathrm{B} 6$ to $\mathrm{C} 7$ region. Thus, although all parts of the mutant CALR C-terminus were immunogenic, the most immunogenic part (the hotspot) was located in the second quartile of the mutant C-terminus.

\section{Cells from healthy subjects display strong, frequent immune responses against peptides spanning the entire mutant CALR C-terminus}

As the B7-C6 hotspot sequence seemed to be highly immunogenic we merged the sequence into one long peptide (CALRLong3) and analyzed the immunogenicity of this epitope. Not surprisingly, 12/14 healthy donors harbored a response to CALRLong3 (Fig. 3a). However, our analysis of the CALR library showed that immune responses are identified agains all parts of the $\mathrm{C}$-terminus. Therefore, we analyzed immune responses against CALRLong4, which spans the 34 most C-terminal amino acids in the mutant $\mathrm{C}$-terminus, and CALRLong36, that spans all 36 amino acids in the CALR-mutant C-terminus. The immunogenicity of the latter was of particular interest, as this peptide is used in the phase I clinical vaccination trial currently running at our institution (NCT03566446). Both CALRLong4 and CALRLong36 incited frequent and strong responses (Fig. 3a). We then performed ELISPOT assays on PBMC plated directly ex vivo and allowed to incubate in the ELISPOT plate for $22 \mathrm{~h}$. Ex vivo responses against CALRLong 4 was found in $4 / 5$ analyzed samples, and three samples displayed a DFR2x-defined significant response (Fig. 3b). Likewise, 2/ 2 analyzed samples showed an ex vivo response against CALRLong36 (Fig. 3c). As the CALRLong4 and CALRLong36 peptides are long peptides and, therefore, need antigen processing for presentation on the cell surface, the $22 \mathrm{~h}$ ex vivo ELISPOT may not show the full response to the mutant epitopes. As such, we performed $72 \mathrm{~h}$ ex vivo IFN- $\gamma$ ELISPOT in PBMC from 11 healthy donors, (Fig. $3 \mathrm{~d})$ and TNF- $\alpha$ ELISPOT in PBMC from ten healthy donors (Fig. 3e). All 11 donors had an IFN- $\gamma$ response, and six displayed a TNF- $\alpha$ response, once more demonstrating that the CALR-mutant epitopes are highly immunogenic, and the responses identified are indeed elicited by circulating CALR-mutant-specific T-cells. By using ICS on in vitro stimulated cultures to investigate the phenotype of the cytokine-producing cells stimulated with CALRLong3, CALRLong4 and CALRLong36 we found that it is mainly $\mathrm{CD} 4^{+} \mathrm{T}$-cells that are activated upon antigen stimulation (Fig. 3f).

\section{The frequency of CALR-mutant-specific immune responses does not change with age}

As the median age of diagnosis of CALR-mutant MPNpatients is $47-57$ years ${ }^{16,19-22}$, we wondered if the emergence of CALR-mutant MPN could be explained by an age dependent loss of CALR-mutant specific immune response. Hence, we analyzed the specific responses against CALRLong1 and CALRLong4 in PBMC from 25 healthy individuals with a median age of 19 years (range 18-21 years) and in PBMC from 25 healthy individuals with a median age of 57 years (range 50-64 years). No difference in frequency nor amplitude of responses against CALRLong1 and CALRLong4 was identified between the two age defined cohorts (Supplementary Material 5A, 5B).

In total we have analyzed the CALR- mutant specific immune responses in a vast amount of healthy subjects: Of 74 subjects analyzed for response against CALRLong1, 54 (78\%) exhibited a DFR-defined significant immune response, and 24 (32\%) exhibited a DFR2x-defined significant response. The responses against CALRLong4 were even more frequent; of 110 subjects analyzed, PBMC from 98 (89\%) exhibited a DFR-defined significant response and 60 individuals (55\%) exhibited a DFR2xdefined significant response.

\section{CALR-mutant specific immune responses are identified in $\mathrm{CD4}^{+} \mathrm{T}_{\text {mem }}$, but not $\mathrm{T}_{\text {naive }}$ cells}

Given the high frequencies and amplitudes of CALRmutant-specific $\mathrm{T}$-cell responses in healthy individuals, we speculated that these $T$-cell responses might be attributable to $\mathrm{T}_{\text {mem }}$ rather than $\mathrm{T}_{\text {naive. }}$. To investigate this hypothesis, we isolated $\mathrm{CD} 4^{+} \mathrm{T}_{\text {mem }}$ from a healthy individual that had shown a clear CALRLong4-specific T-cell response. In these $\mathrm{CD} 4^{+} \mathrm{T}_{\text {mem }}$ enriched cells, we readily detected a strong in vitro immune response against CALRLong4 (Fig. 4a). Similarly, we isolated enriched $\mathrm{T}_{\text {naive }}$ from the same donor, and these cells did not show a 

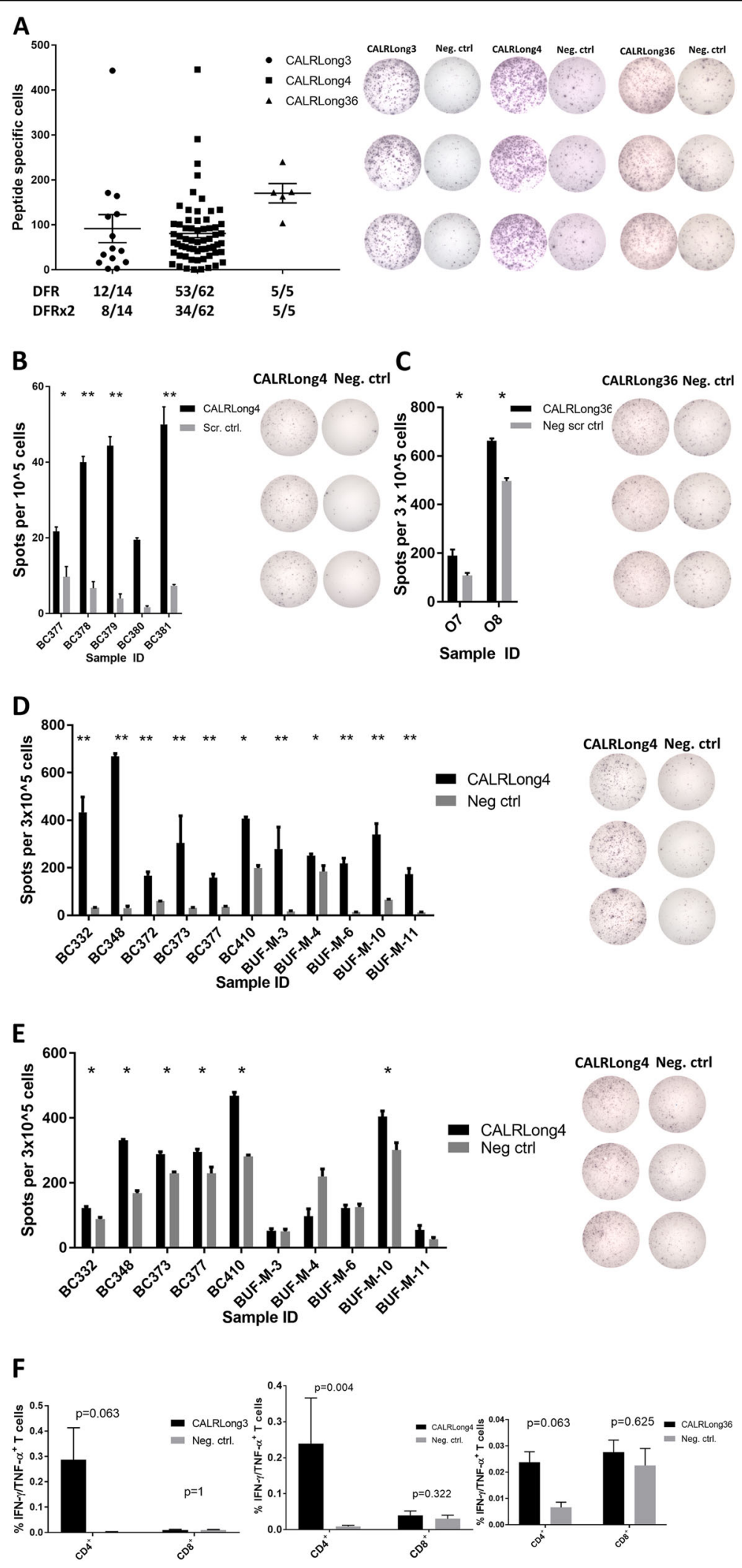

Fig. 3 (See legend on next page.) 
(see figure on previous page)

Fig. 3 In vitro and ex vivo immune responses against epitopes that spanned the entire mutant C-terminus. a (Left) In vitro IFN-y ELISPOT results show the number of cells that specifically responded to CALRLong3, CALRLong4, and CALRLong36 peptides. The DFR-defined and DFR2xdefined responses are indicated below the graph. Example of responses against CALRLong3 (left), CALRLong4 (middle), and CALRLong36 (right). b (Left) Ex vivo $22 \mathrm{~h} \mathrm{IFN- \gamma} \mathrm{ELISPOT} \mathrm{responses} \mathrm{against} \mathrm{CALRLong4} \mathrm{in} \mathrm{cells} \mathrm{from} \mathrm{five} \mathrm{healthy} \mathrm{individuals;} \mathrm{(right)} \mathrm{example} \mathrm{of} \mathrm{a} \mathrm{response.} \mathbf{c}$ (Left) Ex vivo $22 \mathrm{~h}$ IFN- $\gamma$ ELISPOT responses against CALRLong36 in cells from two healthy individuals. d Ex vivo $72 \mathrm{~h}$ IFN- $\gamma$ ELISPOT against CALRLong4 in cells from 11 healthy individuals, with wells displaying a representative response shown to the right. e Ex vivo $72 \mathrm{~h}$ TNF-a ELISPOT against CALRLong4 in cells from ten healthy individuals, with wells displaying a representative response shown to the right. $\mathbf{f}$ Intracellular cytokine staining of cells from healthy individuals depicts IFN- $\gamma / T$ NF-a-double-positive CD4 ${ }^{+}$T-cells stimulated with either CALRLong3 (left), CALRLong4 (middle), or CALRLong36 (right). Cells from ten healthy donors were analyzed for responses against CALRLong4, and cells from five healthy donors were analyzed for response against CALRLong3 and CALRLong5. Error bars display standard error of the mean. ${ }^{*} p \leq 0.05$ according to the DFR rule. ${ }^{* *} p \leq 0.05$ according to the DFR2x rule

specific response to CALRLong4 in vitro (Fig. 4b). However, we detected a CALRLong4-specific immune response in the unsorted PBMC fraction and in the $\mathrm{T}_{\text {naive }}{ }^{-}$ depleted fraction. Next, we used FACS to enrich PBMC from two healthy donors for $\mathrm{T}$ effector memory $\mathrm{T}$-cells $\left(\mathrm{T}_{\mathrm{EM}}\right)$, which were stimulated ex vivo in ELISPOT with CALRLong4. Strong ex vivo immune responses in the highly pure $\mathrm{T}_{\mathrm{EM}}$ cell fractions were detected (Fig. 4c).

\section{Ex vivo CALR-mutant-specific immune responses reside primarily in the $\mathrm{CD}^{+} \mathrm{T}_{\text {mem }}$ compartment}

As the use of FACS for sorting $\mathrm{T}_{\mathrm{EM}}$ cells can potentially interfere with cell functionality, we looked further into the CALR-mutant-specific T-cell responses by sorting PBMCs from three healthy individuals using MACS to enrich for $\mathrm{CD}^{+}{ }^{+} \mathrm{T}_{\text {mem }}$ and $\mathrm{T}_{\text {naive }}$. The enrichments were based on negative selection; i.e., the cells of interest were not treated with antibodies that could either activate or inactivate the cells. We investigated the enriched $\mathrm{T}$ cells with an ex vivo IFN- $\gamma$ ELISPOT assay for responses against CALRLong1, CALRLong4, and CALRLong36. The $\mathrm{CD}_{4}{ }^{+} \mathrm{T}_{\text {mem }}$ from all donors displayed strong CALRmutant-specific $\mathrm{T}$-cell responses, mainly against CALRLong4 and CALRLong36. In samples from two individuals, the enriched $T_{\text {naive }}$ fraction did not display any immune responses (Fig. 5a, b). However, in one individual, both the CD4 ${ }^{+} \mathrm{T}_{\text {mem }}$ fraction and the $\mathrm{T}_{\text {naive }}$ fraction displayed responses against the three mutant epitopes (Fig. 5c). However, the strongest immune response was identified in the $\mathrm{T}_{\text {mem }}$ fraction.

To clarify whether these results might be reproducible by using another enrichment procedure, we performed FACS live-cell sorting to isolate either $\mathrm{T}_{\text {mem }}\left(\mathrm{CD}^{+}\right.$, $\mathrm{CD}^{2} \mathrm{~L}^{+}, \mathrm{CD}^{2} 5 \mathrm{RA}^{-}$; $\mathrm{CD}^{+}, \mathrm{CD}^{-} 2 \mathrm{~L}^{-}, \mathrm{CD}^{-} 5 \mathrm{RA}^{+}$; and $\left.\mathrm{CD}^{+}, \mathrm{CD} 6 \mathrm{~L}^{-}, \mathrm{CD}^{-} 5 \mathrm{RA}^{-}\right)$or $\mathrm{T}_{\text {naive }}\left(\mathrm{CD}^{+}, \mathrm{CD} \mathrm{L}^{+}\right.$, $\mathrm{CD} 4 \mathrm{RA}^{+}$) fractions. Next, we analyzed the enriched fractions and the unsorted cells using ex vivo IFN- $\gamma$ ELISPOT to identify spontaneous immune responses against CALRLong4. Once more, we detected responses in enriched $\mathrm{T}_{\text {mem }}$, whereas no significant responses were detected in the $T_{\text {naive }}$ fraction (Fig. 6a). To determine whether the cryopreservation of PBMC might have influenced the responses, we isolated PBMC from freshly drawn blood from a healthy individual with a known response to CALRLong4. The PBMC were rested overnight and then live-sorted with FACS and assayed in an ex vivo IFN- $\gamma$ ELISPOT. Upon stimulation with CALRLong4 and CALRLong36, we once more detected cytokine release from $T_{\text {mem }}$, but not from $T_{\text {naive }}$ (Fig. 6b). Next, $T_{\text {mem }}$ from the cell sorting were stimulated in vitro for 7 days with the CALRLong4 peptide and analyzed with ICS to investigate the phenotype of the responding $T_{\text {mem }}$. Accordingly, we demonstrated that both $\mathrm{CD}_{4}^{+}$and $\mathrm{CD} 8^{+}$ $\mathrm{T}_{\text {mem }}$ responded to stimulation with CALRLong4 (Fig. 6c). Due to the low yield of $T_{\text {naive }}$ from the cell sorting procedure, we did not analyze potential CALRLong4specific responses in $\mathrm{T}_{\text {naive }}$ with ICS.

\section{CALR-mutant-specific immune responses reside primarily in the $T_{E M}$ and $T_{C M}$ fractions}

As the CALR-mutant specific $T$ cells clearly reside within the $\mathrm{T}_{\text {mem }}$ compartment we then examined which of the cells in this compartment are the most reactive to the $C A L R$-mutations. Hence, we used FACS live-cell sorting to isolate four different $\mathrm{CD}^{+}$T-cell fractions: $\mathrm{T}_{\mathrm{EM}}\left(\mathrm{CD}^{+}\right.$, $\left.\mathrm{CD} 2 \mathrm{~L}^{-}, \mathrm{CD}_{45 \mathrm{RA}^{-}}\right)$, $\mathrm{T}$ central memory cells $\left(\mathrm{T}_{\mathrm{CM}}: \mathrm{CD}^{+}\right.$, $\left.\mathrm{CD} 2 \mathrm{~L}^{+}, \mathrm{CD} 4 \mathrm{RA}^{-}\right)$, terminally differentiated memory cells $\left(\mathrm{T}_{\mathrm{EMRA}}: \mathrm{CD}^{+}, \mathrm{CD} 2 \mathrm{~L}^{-}, \mathrm{CD} 4 \mathrm{RA}^{+}\right)$, and $\mathrm{T}_{\text {naive }}$ $\left(\mathrm{CD}^{+}, \mathrm{CD} 6 \mathrm{~L}^{+}, \mathrm{CD} 45 \mathrm{RA}^{+}\right)$. Then, we analyzed CALRLong4-specific immune responses in sorted cells using ex vivo IFN- $\gamma$ ELISPOT assays. Cells from two different individuals were analyzed. The first sample showed the highest antigen-specific immune responses in the $T_{E M}$ and $\mathrm{T}_{\mathrm{EMRA}}$ fractions (Fig. 7a, top). However, due to the scarcity of cells, we only analyzed $\mathrm{T}_{\mathrm{EM}}$ cell responses in duplicate wells and $\mathrm{T}_{\text {EMRA }}$ cell responses in a single well. We also identified some reactivity in the $\mathrm{T}_{\mathrm{CM}}$ fraction. The lowest cytokine release was identified in the $\mathrm{T}_{\text {naive }}$ fraction. Purity analyses of the enriched fractions invariably showed very high purity (Fig. 7a, bottom). Repeating the sorting procedure in another donor we got a higher cell yield and were able to analyze $\mathrm{T}_{\mathrm{EM}}, \mathrm{T}_{\mathrm{CM}}$, and $\mathrm{T}_{\text {naive }}$ fractions in 


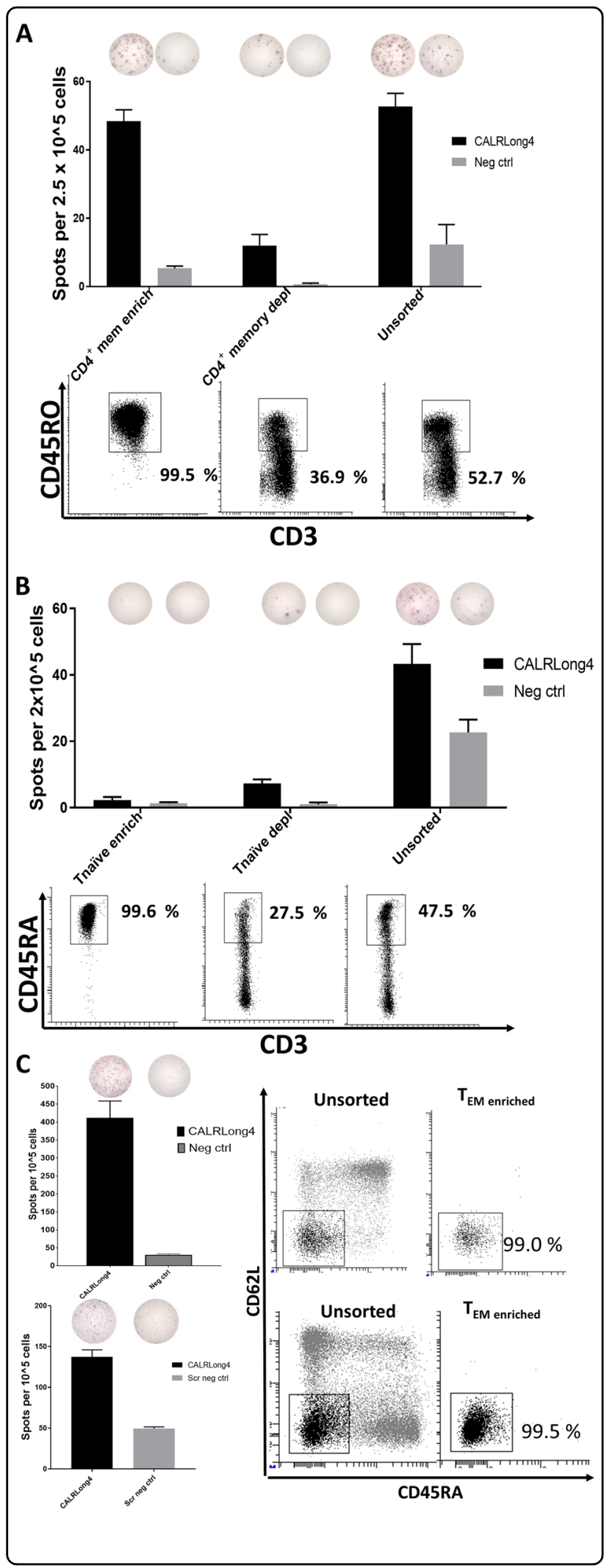

Fig. 4 CALR-mutant-specific T-cell responses in different T-cell compartments. a PBMCs from healthy individuals were enriched for $\mathrm{CD}^{+} \mathrm{T}_{\text {mem }}$ with MACS. Enriched cells, CD4 ${ }^{+} \mathrm{T}_{\text {mem }}$-depleted cells, and unsorted cells were analyzed in a standard IFN- $\gamma$ ELISPOT. (Middle) The number of spots per T-cell fraction and (top) a representative photograph of wells stimulated with either CALRLong4 or a negative scrambled peptide. (Bottom) Purity analyses of $\mathrm{CD}^{+}$gated cells in corresponding cell fractions. ELISPOT assays were performed in triplicate. $\mathbf{b}$ PBMCs from healthy individuals were enriched for $T_{\text {naive }}$ cells with MACS. Enriched cells, Tnaive-depleted cells, and unsorted cells were analyzed in a standard IFN- $-\gamma$ ELISPOT. (Middle) The number of spots per T-cell fraction; (top) a representative photograph of wells stimulated with either CALRLong4 or a negative scrambled peptide. (Bottom) Purity analyses of $\mathrm{CD}^{+}$gated cells in corresponding cell fractions. ELISPOT assays were performed in triplicates. c PBMCs from healthy individuals were enriched for T effector memory cells $\left(T_{E M}\right)$ $\left(\mathrm{CD}^{+}, \mathrm{CD}^{+} 5 \mathrm{RA}^{-}, \mathrm{CD} 6 \mathrm{~L}^{-}\right)$with FACS, and analyzed in an ex vivo IFN- $\gamma$ ELISPOT. (Left) The number of spot-forming cells and a representative photograph of wells stimulated with either CALRLong4 or a negative scrambled peptide. (Right) FACS plots of unsorted PBMCs and $\mathrm{TEM}^{-}$ enriched fractions show the purity of $\mathrm{TEM}_{\mathrm{EM}}$-enriched fractions in $\mathrm{CD}^{+}$ gated cells. Error bars display standard error of the mean

triplicate experiments. Both the $\mathrm{T}_{\mathrm{CM}}$ and $\mathrm{T}_{\mathrm{EM}}$ fractions of this sample displayed very strong CALRLong4-specific immune responses. In contrast, the $\mathrm{T}_{\text {naive }}$ and $\mathrm{T}_{\mathrm{EMRA}}$ responses were very weak (Fig. $7 \mathrm{~b}$ ).

\section{CALR-mutant specific T-cell cultures from CD4 ${ }^{+} \mathrm{T}_{\text {mem }}$ recognize several epitopes from the mutant CALR C- terminus and upregulate CD107a upon antigen stimulation}

We have previously demonstrated that it is possible to isolate and expand CALR-mutant specific $\mathrm{T}$ cells from CALR-mutant MPN-patients. To further verify, that CALRmutant specific $\mathrm{T}$ cells in healthy donors are indeed antigen experienced $\mathrm{T}_{\mathrm{mem}}$, we chose to analyze whether it is possible to isolate and expand CALR-mutant specific $T$ cells from the $\mathrm{T}_{\text {mem }}$ compartment of healthy donors. Hence, using MACS we enriched $C D 4^{+} \mathrm{T}_{\text {mem }}$ from PBMC from two different healthy donors. The cells were enriched twice to ensure a high purity of cells (Fig. 8a, left and 8c, left). Next, the $\mathrm{CD} 4^{+} \mathrm{T}_{\text {mem }}$ were stimulated three times with autologous DCs that were either pulsed with CALRLong1 or CALRLong4 peptide. Antigen responses were measured at day 5 after each DC stimulation using ICS. A total of three DC stimulations were performed. Due to scarcity of T cells, the CALRLong1 specific culture was expanded after the third DC stimulation using our rapid expansion protocol with high dose IL-2. The two T-cell cultures responded with cytokine release (Fig. 8a, c, middle panel), and enhanced expression of the degranulation marker CD107a upon stimulation with antigen (Fig. 8a, c, right panel). This last finding is especially noteworthy, as CALR-mutant specific $\mathrm{T}$ cells from patients were shown to display specific 


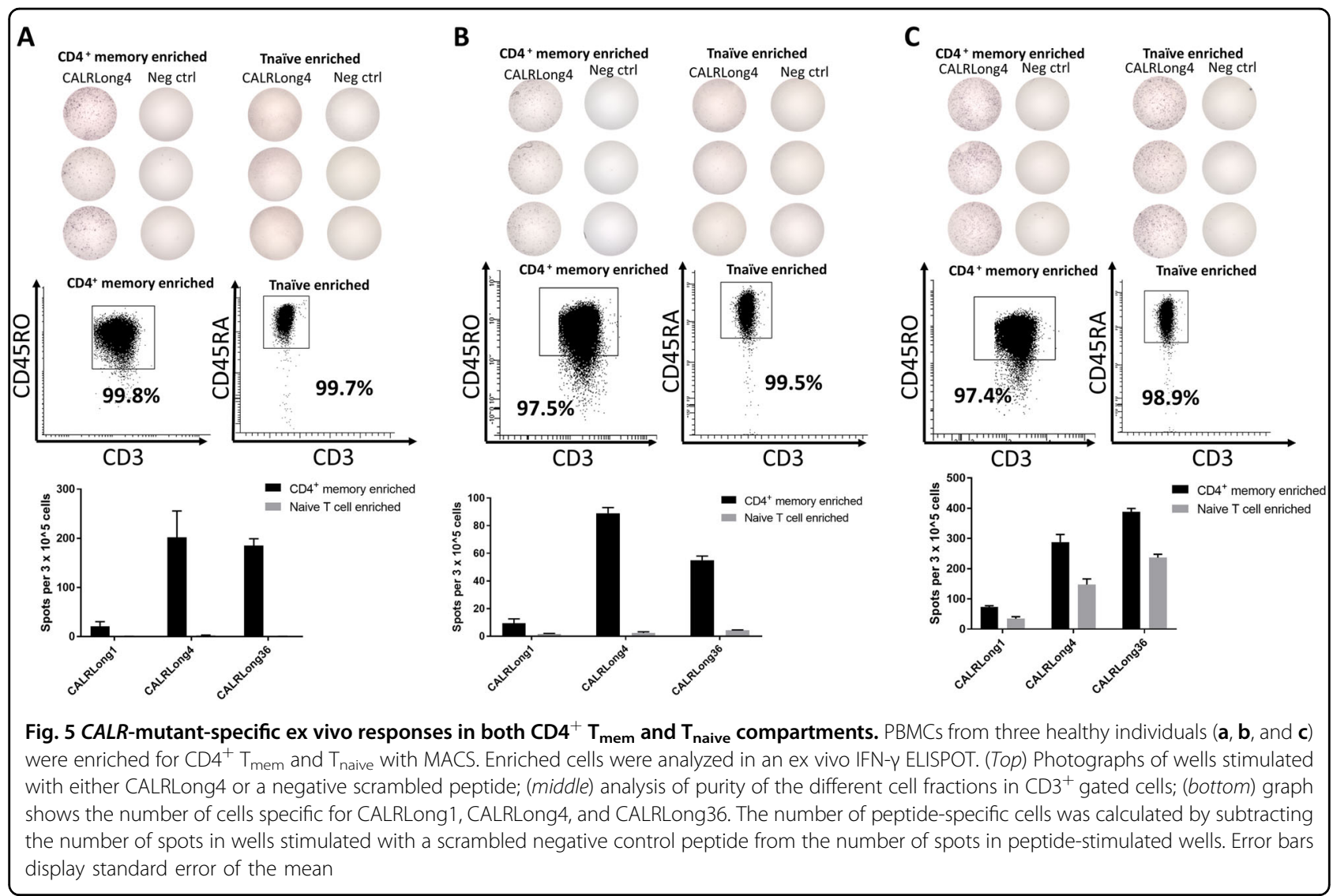

cytotoxicity to autologous CALR-mutant cells through upregulation of CD107a $\mathrm{a}^{4}$. Finally, by analyzing immune responses from the two $\mathrm{T}$-cell cultures, we demonstrated that the CALR-mutant specific $\mathrm{T}$ cells recognize several epitopes in the mutant C-terminus (Fig. 8b, d). Thus, antigen experienced $\mathrm{T}$ cells isolated and expanded from healthy donors do secrete cytokines upon stimulation with several epitopes from the mutant CALR C-terminus, and these $\mathrm{T}$ cells are potentially able to kill $C A L R$-mutant cells.

\section{Discussion}

We previously described in detail that the immune system of CALR-mutant patients specifically reacts to the mutant CALR C-terminus ${ }^{3}$. Even more, we showed that CALR-mutant specific T cells cloned from CALR-mutant patients recognize and kill autologous CALR-mutant target cells in a CALR-mutant-dependent manner ${ }^{4}$. However, in CALRwt individuals, the immune system should not have been challenged with mutant CALR epitopes, and accordingly such individuals should not display a CALR-mutant specific immune response. Surprisingly, we identified CALR-mutant specific immune responses in several JAK2V617F-mutant MPN-patients. Although we did not test whether these patients also carried CALR mutations, we speculated that it would be highly unlikely as $J A K 2$ and CALR double mutants are rare $^{14-16}$. This finding lead us to analyze CALR-mutant specific responses in healthy donors, and we were surprised to find even stronger and more frequent responses in healthy donors compared to the responses identified by us in CALR-mutant MPN-patients ${ }^{3}$. The stronger and more frequent responses might be explained by the theory of cancer immuno-editing; ${ }^{23}$ cancer cells may be cleared by the immune system (in healthy individuals), be held in equilibrium by the immune system (in the MPN-setting; patients with essential thrombocythemia), or escape the immune system and enter into the metastatic disseminated stage (in the MPN-setting; patients with myelofibrosis $)^{23,24}$. By following this notion, the data described here implies, that healthy individuals are able to clear cells harboring the CALR-mutation and, accordingly, this tumor cell elimination generates CALR-mutant specific $\mathrm{T}_{\text {mem. }}$. Interestingly, Tubb et al. $^{25}$ recently isolated CALR-mutant specific $\mathrm{CD}^{+} \mathrm{T}$ cells from peripheral blood in healthy donors using HLA-I tetramers. Even though this study did not provide data on the frequency of healthy donors harboring CALR-mutant specific $\mathrm{T}$ cells, and, most importantly, did not investigate the phenotype of such $\mathrm{T}$ cells, it still supports our findings of immune responses against mutant CALR in healthy individuals. 


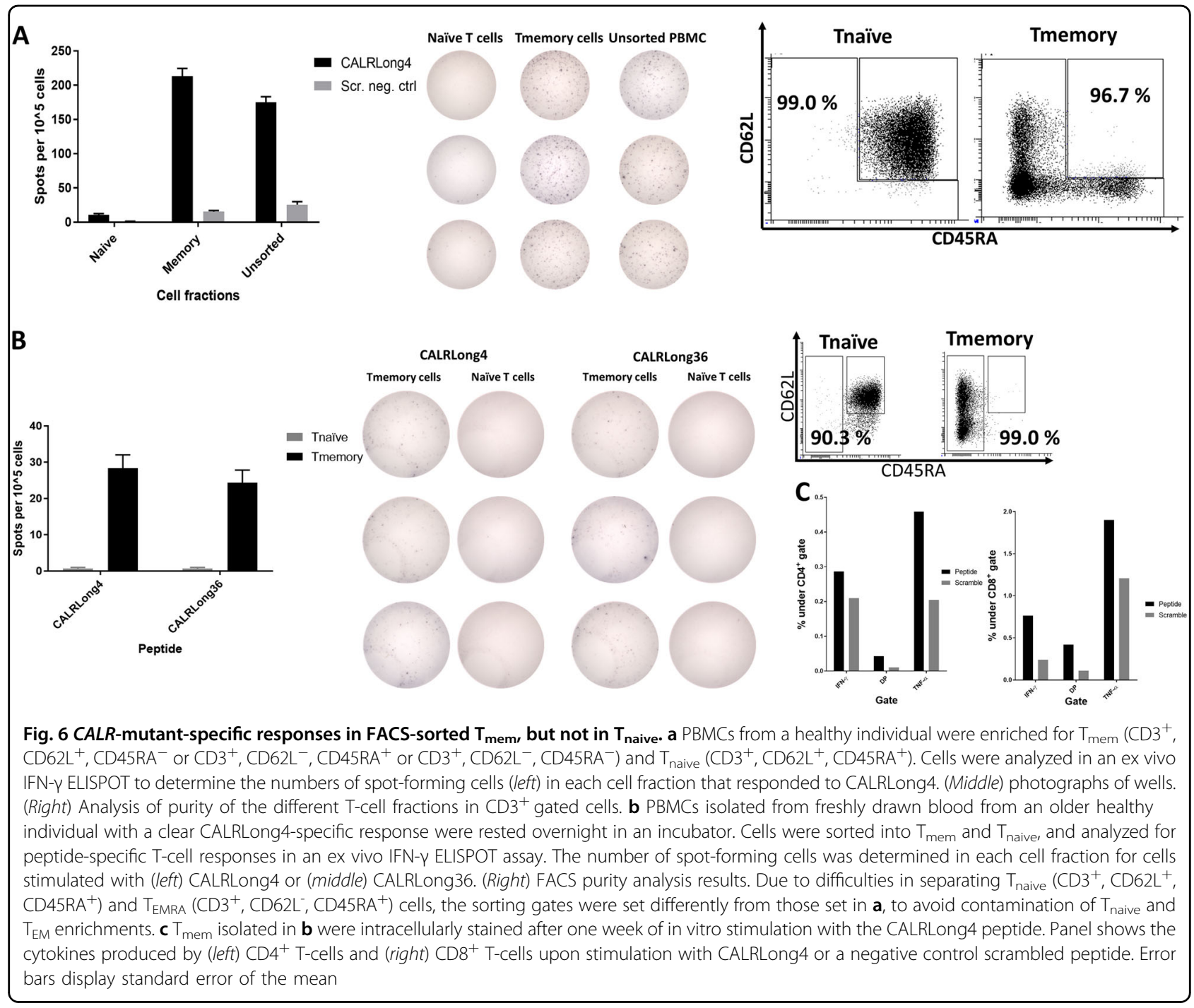

As such, no studies have investigated the phenotype of neo-antigen-specific $\mathrm{T}$ cells in healthy donors, and only a limited number of studies have investigated the memory phenotype of $T$ cells that display reactivity to tumorassociated antigens. Pittet et al. ${ }^{26}$ investigated the phenotype of $\mathrm{CD}^{+} \mathrm{T}$ cells from healthy individuals with a reactivity against the melanoma associated antigen MART-1 and found the specific $T$ cells to be $T_{\text {naive }}$. In contrary we describe by several different means, that $\mathrm{T}_{\text {mem }}$ are activated both in vitro and ex vivo upon stimulation with the mutant CALR antigen. Importantly, we further describe that $\mathrm{T}$-cell cultures derived from high purity $\mathrm{T}_{\mathrm{mem}}$ fractions from two healthy donors reacted against several epitopes in the mutant CALR C-terminal. The data presented in this study imply that the immune system in healthy individuals has been exposed to CALRmutant cells. Given the exquisite immunogenicity of these mutations, the CALR-mutant cells have been cleared by the immune system before the establishment of overt malignancy and, consequently $\mathrm{T}$-cell memory to the $C A L R$-mutations has been generated.

We did not only detect frequent and strong $\mathrm{T}$-cell responses among in vitro stimulated $\mathrm{T}$ cells, but also directly ex vivo. These ex vivo responses are especially remarkable as it, even in cancer patients, is rare to detect tumor-associated antigen-specific T-cells ex vivo by tetramer staining or ELISPOT ${ }^{27}$. As such, it is unusual for cells from healthy individuals to display a high frequency of specific immunity against a cancer neo-antigen.

Given the high frequency of responses to the mutant antigens we verified that the CALR mutations do not share sequence homology with any other known epitope. We also ordered peptides from three different peptide providers (see Materials and methods) in order to rule out the possibility of peptide impurities as an explanation to the T-cell responses. Furthermore, the established T-cell cultures 


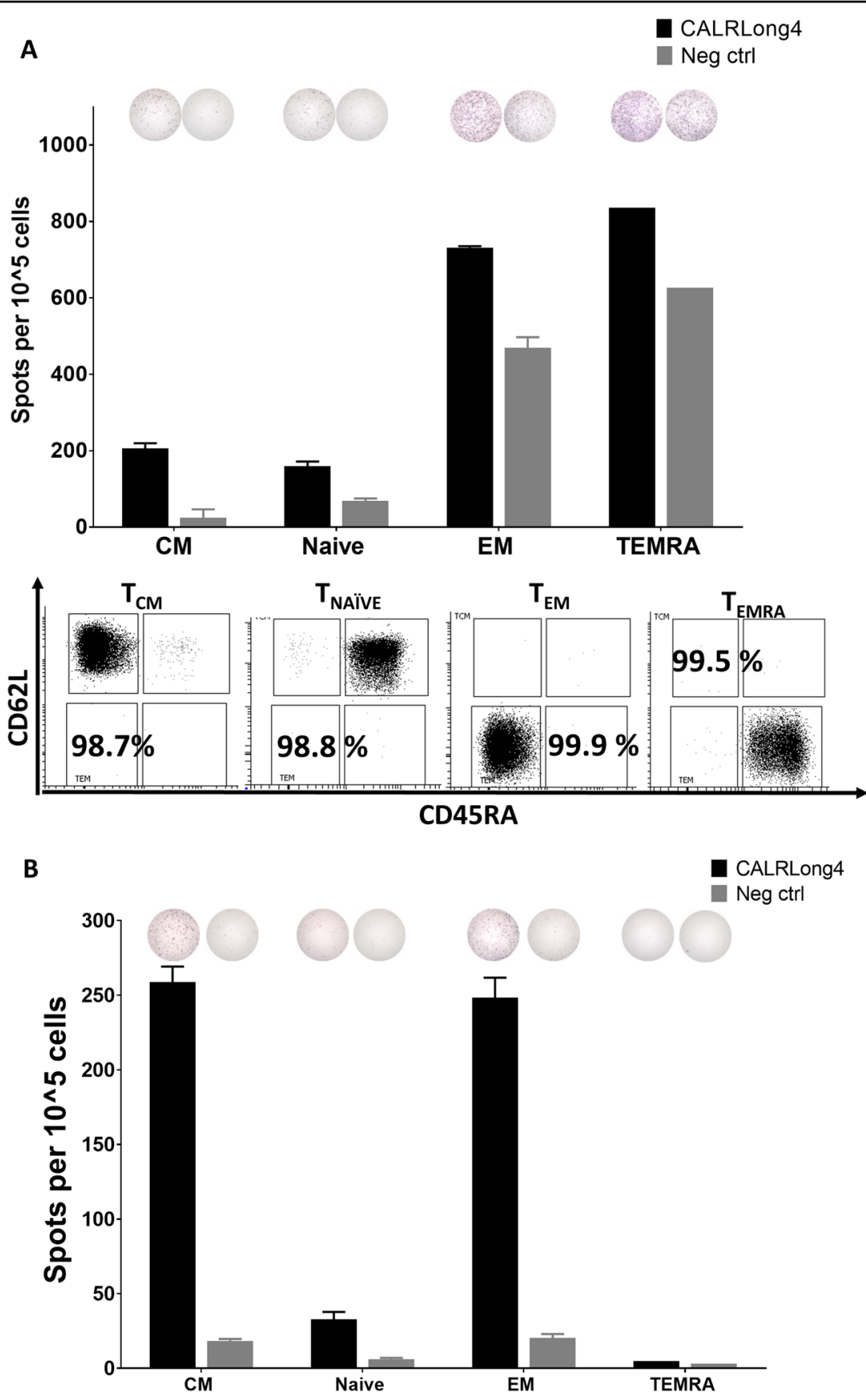

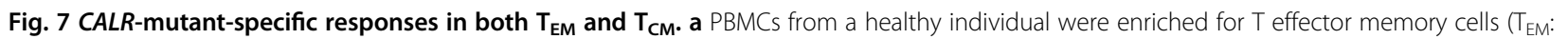

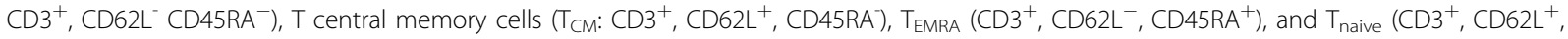
$\mathrm{CD}_{5} \mathrm{RA}^{+}$). Cells were analyzed in an ex vivo IFN- $\mathrm{ELISPOT}$ for responses against CALRLong4. (Top) Representative wells for each cell fraction show responses to both CALRLong4 and a scrambled negative control peptide; (middle) graph shows the numbers of spot-forming cells in each cell fraction. (Bottom) Analysis of purity of the different T-cell fractions in $\mathrm{CD}^{+}$gated cells. Due to cell scarcity, $\mathrm{T}_{\mathrm{EM}}$ and $\mathrm{T}_{\text {EMRA }}$ responses were measured in duplicate and in a single experiment, respectively. $T_{C M}$ and $T_{\text {näive }}$ responses were measured in triplicate. $\mathbf{b}$ PBMCs from healthy individuals were sorted and analyzed as described above. (Top) Representative wells for each cell fraction show responses to both CALRLong4 and a scrambled negative control peptide. (Bottom) Graph shows the number of spot-forming cells in each cell fraction. Due to cell scarcity, we did not perform purity analyses. TEMRA responses were analyzed in a single experiment, but all other cell fractions were analyzed in triplicate. Error bars display standard error of the mean 


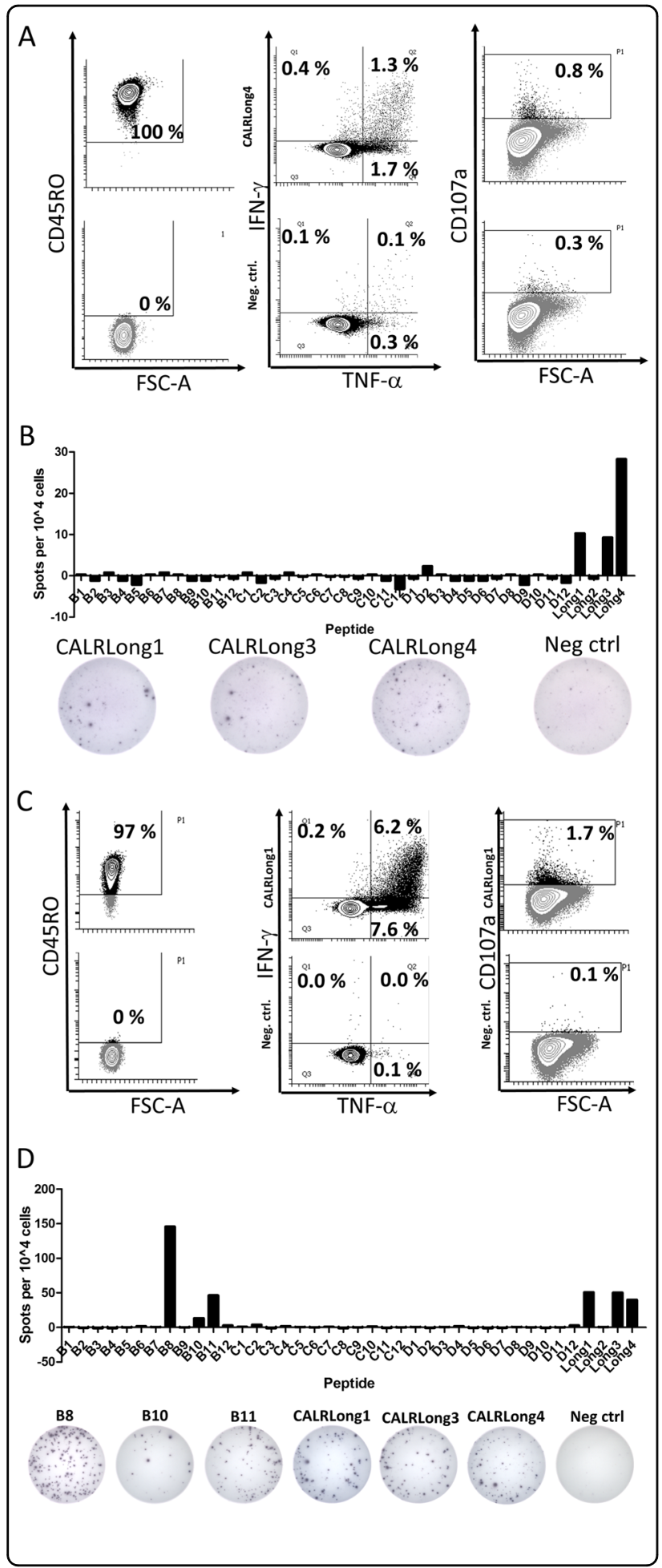

derived from $\mathrm{T}_{\text {mem }}$ recognized several epitopes in the mutant CALR C-terminus, demonstrating that the observed responses indeed are CALR-mutant specific T-cell responses.
Fig. 8 Establishment of two T-cell cultures specific for mutant CALR epitopes. a (Left) Purity analysis of isolated $C D 4^{+}$T-memory cells with cells stained for CD45RO (top, left) and cells that were unstained (below, left). The percentages depict the amount of $\mathrm{CD}_{4} \mathrm{RO}^{+}$cells from the $\mathrm{CD}^{+}$fraction. After three stimulations with autologous DC cells were analyzed for cytokine release against CALRLong4 (top, middle) and scrambled negative control peptide (below, middle). Cells were also analyzed for expression of CD107a upon stimulation with CALRLong4 (top, right) and negative control peptide (below, right). $\mathbf{b}$ The T-cell culture was analyzed for responses against the CALR library and several other CALR-mutant epitopes with the amount of spots shown (top), and representative wells displayed (below). c CD4 ${ }^{+} \mathrm{T}$-memory cells were isolated from another donor and used to establish a T-cell culture specific for the CALRLong1 epitope. Purity analysis (left), cytokine release (middle), and CD107a

upregulation were analyzed as in $\mathbf{a}$. $\mathbf{d}$ The CALRLong1 specific T-cell culture was analyzed for responses against the CALR library. We identified responses against several short epitopes from the CALR

The high frequency of CALR-mutant-specific immune responses observed in healthy individuals samples raises the question why some individuals develop CALR-mutant MPN. The functionality of the immune system is believed to decline with age, and the median age at diagnosis of CALR-mutant MPN is reported to be $47-57$ years ${ }^{16,19-22}$. Therefore, we examined the frequency of immune responses to the CALR-mutant C-terminus in two age defined cohorts (median ages: 19 years vs. age 57 years). However, no differences were found in neither frequency nor response between to two age defined cohorts.

As the CALR mutations are highly immunogenic it might seem paradoxical, that the disease is not cleared by the immune system. However, there are several explanations to the paradoxical emergence of $C A L R$-mutant MPN. Firstly, it was recently shown, that $C A L R$-mutant patients display lower levels of human leukocyte antigen$\mathrm{I}^{28}$, and patients with MPN show increased levels of myeloid derived suppressor cells ${ }^{29}$. Although these explanations could partially clarify why CALR-mutant cells are able to evade immune-mediated destruction, it does not explain why CALR-mutant cells are initially able to establish themselves in the bone marrow, evade immune-mediated destruction, expand and develop overt $C A L R$-mutant MPN. We are currently investigating a cohort of healthy individuals for low-burden CALRmutations and hope that we thereby may identify healthy individuals with low-burden $C A L R$-mutation. A thorough analysis of the immune constitution of such individuals might add important new information on the development and evolution of $C A L R$-mutant MPN, which could potentially be prevented by therapeutic cancer vaccination with CALR-mutant epitopes.

In conclusion, we have demonstrated that a majority of healthy individuals harbor immune cells that display 
CALR-mutant-specific T-cell responses, and that the frequency and amplitude of these responses are higher than in CALR-mutant patients. Neither do the frequency nor amplitude of responses depend on age. Moreover, we show that the majority of the immune responses are attributed to $\mathrm{T}_{\text {mem }}$, particularly cells of the $\mathrm{T}_{\mathrm{EM}}$ and $\mathrm{T}_{\mathrm{CM}}$ compartments. In essence this demonstrates that the immune system in healthy individuals has eliminated tumor cells and generated T-cell memory, thus demonstrating the first of the three E's - elimination - in the theory of cancer immuno-surveillance/editing ${ }^{24}$.

\section{Acknowledgements}

We thank laboratory technician Merete Jonassen for outstanding help teaching M.O.H to perform the immune cell assays. We also thank the students in the third year science class at Himmelev Gymnasium as well as employees at the departments of Hematology at Herlev Hospital and Zealand University Hospital for donating blood for this study. We are also most grateful to Her Royal Highness Crown Princess Mary of Denmark for assisting in development of ELISPOT plates. This study was supported in part by grant from Danish Cancer Society to H.C.H (grant number R149-A10159-B120) and by grants from Region Sjællands Sundhedsvidenskabelige Forskningsfond to M.O.H (grant numbers 12-000095 and 15-000342).

\section{Author details}

'Department of Hematology, Zealand University Hospital, Roskilde, Denmark. ${ }^{2}$ Center for Cancer Immune Therapy, Department of Hematology, Copenhagen University Hospital Herlev, Herlev, Denmark. ${ }^{3}$ Department of Hematology, Rigshospitalet, Copenhagen, Denmark. ${ }^{4}$ Department of Oncology, Copenhagen University Hospital Herlev, Herlev, Denmark. ${ }^{5}$ Faculty of Health and Medical Sciences, University of Copenhagen, Copenhagen, Denmark. ${ }^{6}$ Department of Production, Research, and Innovation, Region Zealand, Sorø, Denmark. ${ }^{7}$ Department of Laboratory Medicine, Boston Children's Hospital, Harvard Medical School, Boston, MA, USA. ${ }^{8}$ Department of Surgical Pathology, Zealand University Hospital, Roskilde, Denmark. ${ }^{9}$ Department of Immunology and Microbiology, University of Copenhagen, Copenhagen, Denmark

\section{Conflict of interest}

It should be noted that Morten Orebo Holmström, Hans Carl Hasselbalch, and Mads Hald Andersen have filed a patent regarding the CALR exon 9 mutations as a target for cancer immune therapy. The patent has been transferred to University Hospital Zealand, Zealand Region and Copenhagen University Hospital at Herlev, Capital Region according to Danish Law concerning inventions made at public research institutions. The other authors declare that they have no conflict of interest.

\section{Publisher's note}

Springer Nature remains neutral with regard to jurisdictional claims in published maps and institutional affiliations.

Supplementary Information accompanies this paper at (https://doi.org/ 10.1038/s41408-018-0166-4).

Received: 23 October 2018 Revised: 26 November 2018 Accepted: 30 November 2018

Published online: 17 January 2019

\section{References}

1. Klampfl, T. et al. Somatic mutations of calreticulin in myeloproliferative neoplasms. N. Engl. J. Med. 369, 2379-2390 (2013).

2. Nangalia, J. et al. Somatic CALR mutations in myeloproliferative neoplasms with nonmutated JAK2. N. Engl. J. Med. 369, 2391-2405 (2013).
3. Holmström, M. O., Riley, C. H., Svane, I. M., Hasselbalch, H. C. \& Andersen, M. H. The CALR exon 9 mutations are shared neoantigens in patients with CALR mutant chronic myeloproliferative neoplasms. Leukemia 30, 2413-2416 (2016).

4. Holmstrom, M. O. et al. The calreticulin (CALR) exon 9 mutations are promising targets for cancer immune therapy. Leukemia 32, 429-437 (2018).

5. Klausen, $U$. et al. Novel strategies for peptide-based vaccines in hematological malignancies. Front. Immunol. 9, 2264 (2018).

6. Kubuschok, B. et al. Naturally occurring T-cell response against mutated p21 Ras oncoprotein in pancreatic cancer. Clin. Cancer Res. 12, 1365-1372 (2006).

7. Somasundaram, R. et al. Human leukocyte antigen-A2-restricted CTL responses to mutated BRAF peptides in melanoma patients. Cancer Res. 66, 3287-3293 (2006).

8. Butt, N. M. et al. Circulating bcr-abl-specific CD8+T cells in chronic myeloid leukemia patients and healthy subjects. Haematologica 90, 1315-1323 (2005).

9. Rusakiewicz, S., Madrigal, A., Travers, P. \& Dodi, A. I. BCR/ABL ${ }^{-}$specific CD8 $+\mathrm{T}$ cells can be detected from CML patients, but are only expanded from healthy donors. Cancer Immunollmmunother. 58, 1449-1457 (2009).

10. Treon, S. P. et al. MYD88 L265P somatic mutation in Waldenström's macroglobulinemia. N. Engl. J. Med. 367, 826-833 (2012).

11. Nielsen, J. S. et al. Mapping the human T cell repertoire to recurrent driver mutations in MYD88 and EZH2 in lymphoma. Oncoimmunology 6, e1321184 (2017).

12. Munir, S. et al. HLA- restricted CTL that are specific for the immune checkpoint ligand PD-L1 occur with high frequency in cancer patients. Cancer Res. 73, 1764-1776 (2013).

13. Moodie, Z. et al. Response definition criteria for ELISPOT assays revisited. Cancer Immunol. Immunother. 59, 1489-1501 (2010).

14. Mcgaffin, G., Harper, K., Stirling, D. \& Mclintock, L. JAK2 V617F and CALR mutations are not mutually exclusive; findings from retrospective analysis of a small patient cohort. Br. J. Haematol. 167, 276-278 (2014).

15. Lundberg, P. et al. Clonal evolution and clinical correlates of somatic mutations in myeloproliferative neoplasms. Blood 123, 2220-2228 (2014).

16. Tefferi, A. et al. CALR vs JAK2 vs MPL mutated or triple-negative myelofibrosis: clinical, cytogenetic and molecular comparisons. Leukemia 28, 1472-1477 (2014).

17. Grinfeld, J. et al. Classification and personalized prognosis in myeloproliferative neoplasms. N. Engl. J. Med. 379, 1416-1430 (2018).

18. National Center for Biotechnology Information, U.S. National Library of Medicine. BLAST: Basic Local Alignment Search Tool. https://blast.ncbi.nlm.nih.gov/ Blast.cgi. https://blast.ncbi.nlm.nih.gov/Blast.cgi (accessed 14 Feb2018).

19. Rumi, E. et al. JAK2 or CALR mutation status defines subtypes of essential thrombocythemia with substantially different clinical course and outcomes. Blood 123, 1544-1551 (2014).

20. Tefferi, A. et al. Long-term sunvival and blast transformation in molecularly annotated essential thrombocythemia, polycythemia vera, and myelofibrosis. Blood 124, 2507-2513 (2014).

21. Andrikovics, $\mathrm{H}$. et al. Distinct clinical characteristics of myeloproliferative neoplasms with calreticulin mutations. Haematologica 99, 1184-1190 (2014).

22. Tefferi, A. et al. Calreticulin mutations and long-term survival in essential thrombocythemia. Leukemia 28, 2300-2303 (2014).

23. Dunn, G. P., Bruce, A. T., Ikeda, H., Old, L. J. \& Schreiber, R. D. Cancer immunoediting: from immuno- surveillance to tumor escape. Nat. Immunol. $\mathbf{3}$, 991-998 (2002).

24. Dunn, G. P., Old, L. J. \& Schreiber, R. D. The three Es of cancer immunoediting. Annu. Rev. Immunol. 22, 329-360 (2004).

25. Tubb, V. M. et al. Isolation of T cell receptors targeting recurrent neoantigens in hematological malignancies. J. Immunother. Cancer 6, 1-14 (2018).

26. Pittet, M. J. et al. High frequencies of naive Melan-A/MART-1-specific CD8(+) $T$ cells in a large proportion of human histocompatibility leukocyte antigen (HLA)-A2 individuals. J. Exp. Med. 190, 705-715 (1999).

27. Keilholz, U. et al. Immunologic monitoring of cancer vaccine therapy: results of a workshop sponsored by the Society for Biological Therapy. J. Immunother. 25, 97-138 (2002).

28. Arshad N., \& Cresswell P. Tumor-associated calreticulin variants functionally compromise the peptide loading complex and impair its recruitment of MHCI. J Biol Chem (2018). https://doi.org/10.1074/jbc.RA118.002836.

29. Wang, J. C. et al. Myeloid-derived suppressor cells in patients with myeloproliferative neoplasm. Leuk. Res. 43, 39-43 (2016). 


\section{QUERY FORM}

\begin{tabular}{|l|c|}
\hline \multicolumn{2}{|c|}{ BLOODCANCERJOURNAL } \\
\hline Manuscript ID & [Art. Id: 166] \\
\hline Author & \\
\hline Editor & \\
\hline Publisher & \\
\hline
\end{tabular}

\section{Journal: BLOODCANCERJOURNAL}

Author :- The following queries have arisen during the editing of your manuscript. Please answer by making the requisite corrections directly in the e.proofing tool rather than marking them up on the PDF. This will ensure that your corrections are incorporated accurately and that your paper is published as quickly as possible.

\begin{tabular}{|c|l|l|}
\hline $\begin{array}{c}\text { Query } \\
\text { No. }\end{array}$ & Description & Author's Response \\
\hline & No queries & \\
\hline
\end{tabular}

